\title{
COINCIDENCE AND INVARIANT APPROXIMATION THEOREMS FOR GENERALIZED $f$-NONEXPANSIVE MULTIVALUED MAPPINGS
}

\author{
A. R. KHAN, F. AKBAR, N. SULTANA, AND N. HUSSAIN \\ Received 22 July 2005; Revised 30 January 2006; Accepted 28 February 2006
}

The main purpose of this paper is to prove some new coincidence and common fixed point theorems for noncommuting generalized $f$-nonexpansive multivalued mappings on non-starshaped domain in the framework of a Banach space. As applications, related common fixed point, invariant approximation, and random coincidence point results are established. This work provides extension as well as substantial improvement of several results in the existing literature.

Copyright (c) 2006 Hindawi Publishing Corporation. All rights reserved.

\section{Introduction and preliminaries}

Let $M$ be a subset of a normed space $(X,\|\cdot\|)$. We denote by $2^{X}, C(X), C B(X)$, and $K(X)$, the families of all nonempty, nonempty closed, nonempty closed bounded, and nonempty compact subsets of $X$, respectively. On $C(X)$, we define the Hausdorff metric $H[22]$, by setting for $A, B \in C(X)$,

$$
H(A, B)=\max \left\{\sup _{a \in A} d(a, B), \sup _{b \in B} d(b, A)\right\}
$$

where $d(a, B)=\inf \{d(a, x): x \in B\}$.

The set $P_{M}(u)=\{x \in M:\|x-u\|=\operatorname{dist}(u, M)\}$ is called the set of best approximants to $u \in X$ from $M$. The diameter of $\mathrm{M}$ is denoted and defined by $\delta(M)=\sup \{\|x-y\|$ : $x, y \in M$ \}. A mapping $f: X \rightarrow X$ has diminishing orbital diameters (d.o.d.) [12] if for each $x \in X, \delta(O(x))<\infty$ and whenever $\delta(O(x))>0$, there exists $n=n_{x} \in \mathbb{N}$ such that $\delta(O(x))>\delta\left(O\left(f^{n}(x)\right)\right)$ where $O(x)=\left\{f^{k}(x): k \in \mathbb{N} \cup\{0\}\right\}$ is the orbit of $f$ at $x$ and $O\left(f^{n}(x)\right)=\left\{f^{k}(x): k \in \mathbb{N} \cup\{0\}\right.$ and $\left.k \geq n\right\}$ is the orbit of $f$ at $f^{n}(x)$ for $n \in \mathbb{N} \cup\{0\}$. We denote the boundary of $M$ by $\partial M$.

Let $f: M \rightarrow M$ be a mapping. A mapping $T: M \rightarrow C(M)$ is called $f$-Lipschitz if, for any $x, y \in M$, there exists $k \geq 0$ such that $H(T x, T y) \leq k d(f x, f y)$. If $k<1$ (resp., $k=1$ ), then $T$ is called $f$-contraction (resp., $f$-nonexpansive). The map $T$ is said to be an 
$f$-nonexpansive-type mapping $[6,19]$ if given $x \in M$ and $u_{x} \in T x$, there is a $u_{y} \in T y$ for each $y \in M$ such that $d\left(u_{x}, u_{y}\right) \leq d(f x, f y)$. $T$ is said to be *-nonexpansive (cf. $[3,6,34]$ if for all $x, y$ in $M$ and $u_{x} \in T x$ with $d\left(x, u_{x}\right)=d(x, T x)$, there exists $u_{y} \in T y$ with $d\left(y, u_{y}\right)=d(y, T y)$ such that $d\left(u_{x}, u_{y}\right) \leq d(x, y)$. The set of fixed points of $T$ (resp., $f$ ) is denoted by $F(T)$ (resp., $F(f)$ ). A point $x \in M$ is a coincidence point (common fixed point) of $f$ and $T$ if $f x \in T x(x=f x \in T x)$. The set of coincidence points of $f$ and $T$ is denoted by $C(f, T)$. The pair $\{f, T\}$ is called commuting if $T f x=f T x$ for all $x \in M$. The pair $\{f, T\}$ is called $R$-weakly commuting [27] if for all $x \in M, f T x \in C(M)$ and there exists $R>0$ such that $H(f T x, T f x) \leq R \operatorname{dist}(f x, T x)$. The pair $\{f, T\}$ is called compatible [14] if $\lim _{n} H\left(T f x_{n}, f T x_{n}\right)=0$ when $\left\{x_{n}\right\}$ is a sequence such that $\lim _{n} f x_{n}=t \in \lim _{n} T x_{n}$ for some $t$ in $M$. The pair $\{f, T\}$ is called nontrivially compatible [12] if $f$ and $T$ are compatible and do have a coincidence point. The pair $\{f, T\}$ is called weakly compatible if they commute at their coincidence points, that is, if $f T x=T f x$ whenever $x \in C(f, T)$. Let $T: M \rightarrow C(M)$. The mapping $f: M \rightarrow M$ is said to be $T$-weakly commuting if for all $x \in M, f f x \in T f x$. If the pair $\{f, T\}$ is weakly compatible, then $f$ is $T$-weakly commuting. However, the converse is not true, in general. If $T$ is single-valued, then $T$-weak commutativity at the coincidence points is equivalent to the weak compatibility (see [14]). The mappings $f$ and $T$ are said to satisfy property (E.A) [14], if there exist a sequence $\left\{x_{n}\right\}$ in $X$, some $a \in X$ and $A \in C(X)$ such that $\lim f x_{n}=a \in A=\lim T x_{n}$. The set $M$ is called $q$-starshaped with $q \in M$ if the segment $[q, x]=\{(1-k) q+k x: 0 \leq k \leq 1\}$ joining $q$ to $x$ is contained in $M$ for all $x \in M$. Suppose that $M$ is $q$-starshaped with $q \in F(f)$ and is both $T$ - and $f$-invariant. Then $T$ and $f$ are called $R$-subweakly commuting on $M$ (see [27]) if for all $x \in M, f T x \in C B(M)$ and there exists a real number $R>0$ such that $H(f T x, T f x) \leq R \operatorname{dist}\left(f x, T_{\lambda} x\right)$ for each $\lambda \in[0,1]$, where $T_{\lambda} x=(1-\lambda) q+\lambda T x$. It is well known that $R$-subweakly commuting maps are $R$-weakly commuting and $R$-weakly commuting maps are compatible and compatible maps are weakly compatible. Howevere, the converse is not true, in general (see $[11,12,14,27]$ ).

A set $M$ is said to have property $(N)[21]$, if

(1) $T: M \rightarrow C(M)$,

(2) $\left(1-k_{n}\right) q+k_{n} T x \subseteq M$, for some $q \in M$ and a fixed sequence of real numbers $k_{n}\left(0<k_{n}<1\right)$ converging to 1 and for each $x \in M$.

Each $q$-starshaped set has the property $(N)$ with respect to any map $T: M \rightarrow C(M)$ but the converse does not hold, in general (see $[8,10])$.

A Banach space $X$ satisfies Opial's condition if for every sequence $\left\{x_{n}\right\}$ in $X$ weakly convergent to $x \in X$, the inequality

$$
\liminf _{n \rightarrow \infty}\left\|x_{n}-x\right\|<\liminf _{n \rightarrow \infty}\left\|x_{n}-y\right\|
$$

holds for all $y \neq x$. Every Hilbert space and the space $l_{p}(1<p<\infty)$ satisfy Opial's condition. The map $T: M \rightarrow C(X)$ is said to be demiclosed at 0 if for every sequence $\left\{x_{n}\right\}$ in $M$ and $\left\{y_{n}\right\}$ in $X$ with $y_{n} \in T x_{n}$ such that $\left\{x_{n}\right\}$ converges weakly to $x$ and $\left\{y_{n}\right\}$ converges to $0 \in X$, then $0 \in T x$. A mapping $T: M \rightarrow C(X)$ is called upper (resp., lower) semicontinuous if for any closed (resp., open) subset $B$ of $X, T^{-1}(B)=\{x \in M: T(x) \cap B \neq \varnothing\}$ is closed (resp., open). If $T$ is both upper and lower semicontinuous, then $T$ is called a continuous map. If $T x \in K(X)$ for all $x \in M$, then $T$ is continuous if and only if $T$ 
is continuous from $M$ into the metric space $(K(X), H)$, where $H$ is the Hausdorff metric induced by the metric $d$. The mapping $T: M \rightarrow C(X)$ is called condensing if for any bounded subset $B$ of $M$ with $\alpha(B)>0, \alpha(T(B))<\alpha(B)$, where $\alpha(B)=\inf \{\epsilon>0: B$ can be covered by a finite number of sets of diameter $\leq \epsilon\}$. The mapping $T: M \rightarrow C(X)$ is called hemicompact if each sequence $\left\{x_{n}\right\}$ in $M$ has a convergent subsequence whenever $d\left(x_{n}, T x_{n}\right) \rightarrow 0$ as $n \rightarrow \infty$. The mapping $T$ is said to satisfy condition (A) (cf. [29]) if for any sequence $\left\{x_{n}\right\}$ in $M, D \in C(M)$ such that $\operatorname{dist}\left(x_{n}, D\right) \rightarrow 0$ and $d\left(x_{n}, T x_{n}\right) \rightarrow 0$ as $n \rightarrow \infty$, then there exists $y \in D$ with $y \in T y$. It is clear that every continuous hemicompact map satisfies condition $(A)$. We also note that condition $(A)$ is always satisfied by continuous condensing mappings. Let $f: M \rightarrow X$ be a mapping. Then $f$ and $T$ are said to satisfy the condition $\left(A^{0}\right)$ (cf. [29]) if for any sequence $\left\{x_{n}\right\}$ in $M, D \in C(M)$ such that $\operatorname{dist}\left(x_{n}, D\right) \rightarrow 0$ and $d\left(f x_{n}, T x_{n}\right) \rightarrow 0$ as $n \rightarrow \infty$, there exists $y \in D$ with $f y \in T y$. The sequence $\left\{x_{n}\right\}$ in $M$ is said to be an asymptotically $T$-regular sequence with respect to $f$ provided $d\left(f x_{n}, T x_{n}\right) \rightarrow 0$ as $n \rightarrow \infty$. If $f=I$ (the identity map on $M$ ), then the sequence $\left\{x_{n}\right\}$ is called asymptotically $T$-regular [22].

Meinardus [20] employed the Schauder fixed point theorem to prove a result regarding invariant approximation. Singh [32] proved the following extension of the result of Meinardus.

Theorem 1.1. Let $T: X \rightarrow X$ be a nonexpansive operator, $M$ a $T$-invariant subset of $X$, and $u \in F(T)$. If $P_{M}(u)$ is nonempty compact and starshaped, then $P_{M}(u) \cap F(T) \neq \varnothing$.

Sahab et al. [23] established the following result which contains Theorem 1.1.

Theorem 1.2. Let $I$ and $T$ be self-maps of $X$ with $u \in F(I) \cap F(T)$ and $M \subset X$ with $T(\partial M)$ $\subset M$, and $q \in F(I)$. If $D=P_{M}(u)$ is compact and $q$-starshaped, $I(D)=D, I$ is continuous and linear on $D$, Iand $T$ are commuting on $D$, and $T$ is $I$-nonexpansive on $D \cup\{u\}$, then $P_{M}(u) \cap F(T) \cap F(I) \neq \varnothing$.

Jungck and Sessa [13] proved the following result in best approximation theory, which extends Theorems 1.1 and 1.2 and many others.

Theorem 1.3. Let $I$ and $T$ be selfmaps of a Banach space $X$ with $u \in F(I) \cap F(T)$ and $M \subset$ $X$ with $T(\partial M) \subset M$. Suppose that $D=P_{M}(u)$ is q-starshaped with $q \in F(I), I(D)=D, I$ is affine and continuous in the weak and strong topology on D. If I and T are commuting on $D$ and $T$ is $I$-nonexpansive on $D \cup\{u\}$, then $P_{M}(u) \cap F(T) \cap F(I) \neq \varnothing$ provided either (i) $D$ is weakly compact and $(I-T)$ is demiclosed, or (ii) $D$ is weakly compact and $X$ satisfies Opial's condition.

Recently, Al-Thagafi [2] extended Theorem 1.2 and proved some results on invariant approximations for commuting maps. More recently, Shahzad [24-28], Hussain and Khan [9], and Hussain et al. [10] have further extended and improved the above-mentioned results for noncommuting maps. Latif and Tweddle [18] have proved some coincidence and common fixed point theorems for a commuting pair of $f$-nonexpansive multivalued mappings defined on starshaped subsets of a Banach space. In [27], Shahzad has obtained extension of the results of Latif and Tweddle [18] for $R$-subweakly commuting $f$-nonexpansive maps. Recently, Hussain [8] proved the above-mentioned coincidence 
point results without any type of commutativity of the maps defined on a non-starshaped domain.

The aim of this paper is to first improve and extend the above-mentioned coincidence point results; in particular, we replace the $f$-nonexpansiveness of $T$ by generalized $f$-nonexpansiveness and starshapedness of the set $M$ by a weaker set of conditions. Afterwards, we study the existence of common fixed points of a general class of noncommuting generalized $f$-nonexpansive mappings. As applications, results regarding $*-$ nonexpansive- and $f$-nonexpansive-type maps are derived and invariant approximation and random coincidence results are proved. These results improve and extend the recent results of Husain and Latif [6, 7], Liu et al. [19], Xu [33, 34], Jungck and Sessa [13], Kamran [14], Latif and Bano [17], Sahab et al. [23], Shahzad [24-30], and many others. Several examples are presented which show that certain hypotheses of our results cannot be relaxed.

The following coincidence point result is a consequence of [22, Theorem 3] of Pathak and Khan, which will be needed for the main results.

Theorem 1.4. Let $(X, d)$ be a metric space and let $f: X \rightarrow X$ and $T: X \rightarrow C(X)$ be such that $T(X) \subset f(X)$. Assume that $T(X)$ or $f(X)$ is complete and $T$ and $f$ satisfy for all $x, y \in X$ and $0 \leq h<1$,

$$
\begin{gathered}
H(T x, T y) \leq h \max \{d(f x, f y), \operatorname{dist}(f x, T x), \operatorname{dist}(f y, T y), \\
\left.\frac{1}{2}[\operatorname{dist}(f x, T y)+\operatorname{dist}(f y, T x)]\right\} .
\end{gathered}
$$

Then $C(f, T) \neq \varnothing$.

Lemma 1.5 [18, Lemma 2.2]. Let $X$ be a Banach space which satisfies Opial's condition and $M$ a nonempty weakly compact subset of $X$. Let $f: M \rightarrow X$ be a weakly continuous mapping and $T: M \rightarrow K(X)$ an $f$-nonexpansive map. Then $f-T$ is demiclosed.

The following general common fixed point result is a consequence of [12, Corollary 3.13].

Theorem 1.6. Let $(X, d)$ be a metric space and $g$ a continuous self-map of $X$. If $g$ has relatively compact orbits with d.o.d., then $g$ has a fixed point. Moreover, if $f$ is continuous and the pair $\{f, g\}$ is nontrivially compatible, then there exists a point $z$ in $X$ such that $f z=g z=z$.

Theorem 1.7 [29, Theorem 3.1]. Let $M$ be a nonempty separable weakly compact subset of a Banach space $X$ and $f: \Omega \times M \rightarrow M$ a random operator which is both continuous and weakly continuous. Assume that $T: \Omega \times M \rightarrow C B(M)$ is a continuous random operator such that $(f-T)(\omega, \cdot)$ is demiclosed at 0 for each $\omega \in \Omega$. If $f$ and $T$ have a deterministic coincidence point, then $f$ and Thave a random coincidence point.

Theorem 1.8 [29, Theorem 3.12]. Let $M$ be a nonempty separable complete subset of a metric space $X$ and let $T: \Omega \times M \rightarrow C(X)$ and $f: \Omega \times M \rightarrow X$ be continuous random operators 
A. R. Khan et al. 5

satisfying condition $\left(A^{0}\right)$. If $f$ and Thave a deterministic coincidence point, then $f$ and $T$ have a random coincidence point.

\section{Coincidence and common fixed point results}

The following result extends and improves [18, Theorem 2.1] and [27, Theorem 2.1], in the sense that the maps $f$ and $T$ need not be commuting or $R$-subweakly commuting, $T$ is not necessarily $f$-nonexpansive, $f$ is not affine and continuous, and $M$ is not necessarily $q$-starshaped.

Theorem 2.1. Let $f$ be a selfmap on a nonempty complete subset $M$ of a normed space $X$ such that $f(M)=M$. Assume that $T: M \rightarrow C(M)$ satisfies, for all $x, y \in M$ and $\lambda \in[0,1]$,

$$
\begin{gathered}
H(T x, T y) \leq \max \left\{\|f x-f y\|, \operatorname{dist}\left(f x, T_{\lambda} x\right), \operatorname{dist}\left(f y, T_{\lambda} y\right),\right. \\
\left.\frac{1}{2}\left[\operatorname{dist}\left(f x, T_{\lambda} y\right)+\operatorname{dist}\left(f y, T_{\lambda} x\right)\right]\right\} .
\end{gathered}
$$

Suppose that $T(M)$ is bounded and $(f-T)(M)$ is closed. If $M$ has the property $(N)$, then $C(f, T) \neq \varnothing$. If, in addition, $f$ is T-weakly commuting at $v$ and $f f v=f v$ for $v \in C(f, T)$, then $F(f) \cap F(T) \neq \varnothing$.

Proof. Take $q \in M$ and define $T_{n}: M \rightarrow C(M)$ by

$$
T_{n} x=\left(1-k_{n}\right) q+k_{n} T x
$$

for all $x \in M$ and fixed sequence of real numbers $k_{n}\left(0<k_{n}<1\right)$ converging to 1 . Then, for each $n, T_{n}(M) \subset M=f(M)$ and

$$
\begin{gathered}
H\left(T_{n} x, T_{n} y\right) \leq k_{n} H(T x, T y) \leq k_{n} \max \left\{\|f x-f y\|, \operatorname{dist}\left(f x, T_{n} x\right), \operatorname{dist}\left(f y, T_{n} y\right),\right. \\
\left.\frac{1}{2}\left[\operatorname{dist}\left(f x, T_{n} y\right)+\operatorname{dist}\left(f y, T_{n} x\right)\right]\right\}
\end{gathered}
$$

for each $x, y \in M$ and $0<k_{n}<1$. By Theorem 1.4, for each $n \geq 1$, there exists $x_{n} \in M$ such that $f x_{n} \in T_{n} x_{n}$. This implies that there is a $y_{n} \in T x_{n}$ such that $f x_{n}-y_{n}=\left(1-k_{n}\right)(q-$ $\left.y_{n}\right)$. Since $T(M)$ is bounded and $k_{n} \rightarrow 1$, it follows that $f x_{n}-y_{n} \rightarrow 0$ as $n \rightarrow \infty$. As $(f-$ $T)(M)$ is closed so $0 \in(f-T)(M)$. Hence $C(f, T) \neq \varnothing$. If $f$ is $T$-weakly commuting at $v \in C(f, T)$, then $f f v \in T f v$ and hence $f v=f f v \in T f v$. Thus $F(f) \cap F(T) \neq \varnothing$.

Corollary 2.2 [8, Theorem 2.2]. Let $f$ be a selfmap on a nonempty complete subset $M$ of a normed space $X$ with $f(M)=M$. Assume that $T: M \rightarrow C(M)$ is $f$-nonexpansive map such that $T(M)$ is bounded and $(f-T)(M)$ is closed. If $M$ has the property $(N)$, then $C(f, T) \neq \varnothing$.

Clearly, each $T$-invariant $q$-starshaped set satisfies the property $(N)$, if $f$ is affine, then $T_{n}(M) \subset f(M)$ provided $T(M) \subset f(M)$ and $q \in F(f)$; consequently, we obtain the following result which improves substantially [27, Theorem 2.1]. 
Corollary 2.3. Let $M$ be a nonempty q-starshaped subset of a normed space $X$ and $f$ : $M \rightarrow M$ an affine mapping with $q \in F(f)$. Assume that $T: M \rightarrow C(M)$ is $f$-nonexpansive map, $T(M)$ is bounded, $T(M) \subset f(M)$, and $(f-T)(M)$ is closed. If $f(M)$ is complete, then $C(f, T) \neq \varnothing$. If, in addition, $f$ is T-weakly commuting at $v$ and $f f v=f v$ for $v \in C(f, T)$, then $F(f) \cap F(T) \neq \varnothing$.

The conclusion of [18, Theorem 2.2(a)] holds without any type of commutativity of $f$ and $T, T$ need not be $f$-nonexpansive and compact-valued, as follows.

THeOrem 2.4. Let $f$ be a selfmap on a nonempty weakly compact subset $M$ of a Banach space $X$. Assume that $T: M \rightarrow C(M)$ satisfies (2.1), for all $x, y \in M$ and $\lambda \in[0,1]$. If $M=$ $f(M),(f-T)$ is demiclosed at 0 and $M$ has the property $(N)$, then $C(f, T) \neq \varnothing$. If, in addition, $f$ is $T$-weakly commuting at $v$ and $f f v=f v$ for $v \in C(f, T)$, then $F(f) \cap F(T) \neq \varnothing$.

Proof. For each $x \in M, T x \subset M$, therefore $T(M) \subset M$. Now $M$ is bounded (see [18, 27]), so $T(M)$ is bounded. As in the proof of Theorem 2.1, $f x_{n}-y_{n} \rightarrow 0$ as $n \rightarrow \infty$, where $y_{n} \in T x_{n}$. By the weak compactness of $M$, there is a subsequence $\left\{x_{m}\right\}$ of the sequence $\left\{x_{n}\right\}$ such that $\left\{x_{m}\right\}$ converges weakly to $y \in M$ as $m \rightarrow \infty$. Since $(f-T)$ is demiclosed at 0 , we obtain $0 \in(f-T) y$. Thus $C(f, T) \neq \varnothing$. As in the proof of Theorem 2.1, $F(f) \cap$ $F(T) \neq \varnothing$.

The following result extends and improves [27, Theorem 2.2 and Corollary 2.3], [4, Theorem 2], [11, Corollary 3.4], and [18, Theorem 2.2].

Corollary 2.5 [8, Theorems 2.3 and 2.4]. Let $f$ be a selfmap on a nonempty weakly compact subset $M$ of a Banach space $X$. Assume that $T: M \rightarrow C(M)$ is $f$-nonexpansive map such that $M=f(M)$ and $M$ has the property $(N)$. Then $C(f, T) \neq \varnothing$ provided one of the following two conditions is satisfied:

(a) $(f-T)$ is demiclosed at 0 ;

(b) $f$ is weakly continuous, $T$ is compact-valued, and $X$ satisfies Opial's condition.

If, in addition, $f$ is T-weakly commuting at $v$ and $f f v=f v$ for $v \in C(f, T)$, then $F(f) \cap$ $F(T) \neq \varnothing$.

Proof. (a) It follows from Theorem 2.4.

(b) By Lemma 1.5, $(f-T)$ is demiclosed at 0 . Hence the result from part (a).

TheOREM 2.6. Let $M$ be a nonempty complete subset of a normed space $X$ and $f: M \rightarrow M$ a mapping such that $M=f(M)$. Assume that $T: M \rightarrow C(M)$ satisfies (2.1), for all $x, y \in M$ and $\lambda \in[0,1]$. If $M$ has the property $(N), f$ and $T$ satisfy the condition $\left(A^{0}\right)$, and $T(M)$ is bounded, then $C(f, T) \neq \varnothing$. If, in addition, $f$ is T-weakly commuting at $v$ and $f f v=f v$ for $v \in C(f, T)$, then $F(f) \cap F(T) \neq \varnothing$.

Proof. By Theorem 2.1, for each $n$, there are $x_{n} \in M$ and $y_{n} \in T x_{n}$ such that $f x_{n}-y_{n} \rightarrow$ 0 as $n \rightarrow \infty$. It further implies that $d\left(f x_{n}, T x_{n}\right) \rightarrow 0$ as $n \rightarrow \infty$. By the condition $\left(A^{0}\right)$ there exists an $x_{0} \in M$ such that $f x_{0} \in T x_{0}$. Hence $C(f, T) \neq \varnothing$. As in the proof of Theorem 2.1, $F(f) \cap F(T) \neq \varnothing$.

Corollary 2.7. Let $M$ be a nonempty q-starshaped subset of a normed space $X$ and $f$ : $M \rightarrow M$ an affine mapping with $q \in F(f)$. Assume that $T: M \rightarrow C(M)$ is $f$-nonexpansive 
map, $T(M)$ is bounded, $T(M) \subset f(M)$, and $f$ and $T$ satisfy condition $\left(A^{0}\right)$. If $f(M)$ is complete, then $C(f, T) \neq \varnothing$. If, in addition, $f$ is $T$-weakly commuting at $v$ and $f f v=f v$ for $v \in C(f, T)$, then $F(f) \cap F(T) \neq \varnothing$.

If we take $f=I$, the identity map in the above Corollary, then we get the following corollaries which extend and generalize the results of Dotson [4], Habiniak [5], and Lami Dozo [16].

Corollary 2.8. Let $M$ be a nonempty complete subset of a normed space $X$ and $T: M \rightarrow$ $C(M)$ a nonexpansive mapping such that $T(M)$ is bounded. If $M$ has property $(N)$ and $T$ satisfies the condition $(A)$, then $T$ has a fixed point.

Corollary 2.9. Let $M$ be a nonempty complete subset of a normed space $X$ and $T: M \rightarrow$ $C(M)$ a nonexpansive hemicompact (or condensing) mapping. If $M$ has property $(N)$ and $T(M)$ is bounded, then $T$ has a fixed point.

The following theorem extends and improves [18, Theorem 2.3] of Latif and Tweddle and $[4$, Theorem 1] of Dotson.

Theorem 2.10. Let $M$ be a nonempty compact subset of a normed space $X$ and $f: M \rightarrow M$ a continuous map such that $M=f(M)$. Assume that $T: M \rightarrow C B(M)$ satisfies (2.1), for all $x, y \in M$ and $\lambda \in[0,1]$ and $T$ is continuous (or assume that $T$ is $f$-nonexpansive mapping). If $M$ has property $(N)$, then $C(f, T) \neq \varnothing$. If, in addition, $f$ is $T$-weakly commuting at $v$ and $f f v=f v$ for $v \in C(f, T)$, then $F(f) \cap F(T) \neq \varnothing$.

Proof. By Theorem 2.1, $f x_{n}-y_{n} \rightarrow 0$ as $n \rightarrow \infty$. It further implies that $d\left(f x_{n}, T x_{n}\right) \rightarrow 0$ as $n \rightarrow \infty$. Since $M$ is compact, without loss of generality, we may assume that $\left\{x_{n}\right\}$ converges to some $x_{0} \in M$. The continuity of both $f$ and $T$ implies that $f x_{0} \in T x_{0}$. Hence $C(f, T) \neq$ $\varnothing$. As in the proof of Theorem 2.1, $F(f) \cap F(T) \neq \varnothing$.

The following result improves and extends a recent result due to Beg et al. [3].

Theorem 2.11. Let $M$ be a nonempty complete subset of a normed space $X$ and $f: M \rightarrow M$ a mapping such that $M=f(M)$. Assume that $T: M \rightarrow C(M)$ satisfies (2.1), for all $x, y \in M$ and $\lambda \in[0,1]$ (or assume that $T$ is $f$-nonexpansive mapping). If $M$ has property $(N), T(M)$ is bounded, and $T$ and $f$ satisfy for all $x, y \in M$,

$$
H^{r}(T x, T y) \leq \theta_{1}(d(f x, T x)) d^{r}(f x, T x)+\theta_{2}(d(f y, T y)) d^{r}(f y, T y)
$$

where $\theta_{i}: \mathbb{R} \rightarrow[0,1)(i=1,2)$ and $r$ is some fixed positive real number, then $C(f, T) \neq \varnothing$. If, in addition, $f$ is T-weakly commuting at $v$ and $f f v=f v$ for $v \in C(f, T)$, then $F(f) \cap$ $F(T) \neq \varnothing$.

Proof. By Theorem 2.1, $d\left(f x_{n}, T x_{n}\right) \rightarrow 0$ as $n \rightarrow \infty$. Thus $\left\{x_{n}\right\}$ is an asymptotically $T$ regular sequence with respect to $f$ in $M$. By (2.4),

$$
\begin{aligned}
H^{r}\left(T x_{n}, T x_{m}\right) \leq & \theta_{1}\left(d\left(f x_{n}, T x_{n}\right)\right) d^{r}\left(f x_{n}, T x_{n}\right) \\
& +\theta_{2}\left(d\left(f x_{m}, T x_{m}\right)\right) d^{r}\left(f x_{m}, T x_{m}\right)
\end{aligned}
$$


where the right-hand side tends to 0 as $n, m \rightarrow \infty$. This implies that $\left\{T x_{n}\right\}$ is a Cauchy sequence in $T M \subset M$. Hence there exists $K \in C(M)$ such that $H\left(T x_{n}, K\right) \rightarrow 0$ as $n \rightarrow \infty$. Suppose that $k_{0} \in K$ and let $x_{0} \in f^{-1}\left(k_{0}\right)$. Then $f x_{0}=k_{0} \in K$. Again, by (2.4),

$$
\begin{aligned}
d^{r}\left(f x_{0}, T x_{0}\right) & \leq H^{r}\left(K, T x_{0}\right) \leq \lim _{n \rightarrow \infty} H^{r}\left(T x_{n}, T x_{0}\right) \\
& \leq \lim _{n \rightarrow \infty}\left[\theta_{1}\left(d\left(f x_{n}, T x_{n}\right)\right) d^{r}\left(f x_{n}, T x_{n}\right)+\theta_{2}\left(d\left(f x_{0}, T x_{0}\right)\right) d^{r}\left(f x_{0}, T x_{0}\right)\right] \\
& \leq \theta_{2}\left(d\left(f x_{0}, T x_{0}\right)\right) d^{r}\left(f x_{0}, T x_{0}\right),
\end{aligned}
$$

which yields $\left(1-\theta_{2}\left(d\left(f x_{0}, T x_{0}\right)\right)\right) d^{r}\left(f x_{0}, T x_{0}\right) \leq 0$. Thus $d\left(f x_{0}, T x_{0}\right)=0$ implies that $C(f, T) \neq \varnothing$. Now, as in the proof of Theorem 2.1, $F(f) \cap F(T) \neq \varnothing$.

Example 2.12. Let $X=\mathbb{R}$ and $M=\{0,1\}$ be endowed with the usual metric. Define $T$ : $M \rightarrow K(M)$ by $T x=\{0\}$ for each $x \in M$. Clearly, $M$ is not starshaped but $M$ has the property $(N)$ for $q=0, k_{n}=1-1 /(n+1)$. Let $f: M \rightarrow M$ be defined by $f(x)=1-x$ for each $x \in M$. All of the conditions of Theorem 2.10 are satisfied; consequently $T$ and $f$ have a coincidence point. Here [27, Theorems 2.1 and 2.2] and [18, Theorem 2.3] cannot be applied because $f$ and $T$ are not $\mathbb{R}$-weakly commuting (and hence not commuting) and $M$ is not $q$-starshaped.

Example 2.13. Let $X=\mathbb{R}$ and $M=\{0,1,1-1 /(n+1): n \in \mathbb{N}\}$ be endowed with the usual metric. Define $T(1)=\{0\}$ and $T(0)=T(1-1 /(n+1))=\{1\}$ for all $n \in \mathbb{N}$. Clearly, $M$ is not starshaped but $M$ has the property $(N)$ for $q=0$ and $k_{n}=1-1 /(n+1), n \in \mathbb{N}$. Let $f x=x$ for all $x$ in $M$. Now $f$ and $T$ satisfy (2.1) together with all other conditions of Theorem 2.1 except the condition that $(f-T)(M)=\{-1,-1 /(n+1), 1\}$ is closed. Note that $C(f, T)=\varnothing$. Here also note that all of the conditions of Theorem 2.10 are satisfied except the condition that $T$ is continuous. Note that $C(f, T)=\varnothing$.

Example 2.14. Let $X=\mathbb{R}^{2}$ be endowed with the norm $\|\cdot\|$ defined by $\|(a, b)\|=|a|+$ $|b|,(a, b) \in \mathbb{R}^{2}$.

(1) Let $M=A \cup B$, where $A=\{(a, b) \in X: 0 \leq a \leq 1,0 \leq b \leq 4\}$ and $B=\{(a, b) \in X$ : $2 \leq a \leq 3,0 \leq b \leq 4\}$. Define $T: M \rightarrow K(M)$ by

$$
T(a, b)= \begin{cases}\{(2, b)\} & \text { if }(a, b) \in A \\ \{(1, b)\} & \text { if }(a, b) \in B\end{cases}
$$

and $f(x)=x$, for all $x \in M$. All of the conditions of Theorem 2.10 are satisfied except that $M$ has property $(N)$, that is, $\left(1-k_{n}\right) q+k_{n} T(M)$ not contained in $M$ for any choice of $q \in M$ and $k_{n}$. Note that $C(f, T)=\varnothing$.

(2) $M=\{(a, b) \in X: 0 \leq a<\infty, 0 \leq b \leq 1\}$ and $T: M \rightarrow K(M)$ is defined by

$$
T(a, b)=\{(a+1, b)\}, \quad(a, b) \in M,
$$

and $f(x)=x$, for all $x \in M$. All of the conditions of Theorem 2.10 are satisfied except that $M$ is compact. Note that $C(f, T)=\varnothing$. 
Example 2.15. Let $X=\mathbb{R}$ and $M=[0,1]$ be endowed with the usual metric. Define $f$ : $M \rightarrow M$ and $T: M \rightarrow K(M)$ as follows:

$$
f x=\frac{x+1}{2}, \quad T x=\left[0, \frac{x+1}{3}\right], \quad x \in M .
$$

All of the conditions of Theorem 2.10 are satisfied except that $f(M)=M$. Note that $C(f, T)=\varnothing$.

The following example reveals that the condition $f f v=f v$ for $v \in C(f, T)$, in Theorem 2.10 , is necessary for the result.

Example 2.16. Let $X=\mathbb{R}$ and $M=[0,1]$ be endowed with the usual metric. Define $T(x)=$ $\{0,1\}$ and $f(x)=1-x$ for each $x \in M$. All of the conditions of Theorem 2.10 are satisfied except the condition $f f v=f v$ for $v \in C(f, T)$. Note that $F(f) \cap F(T)=\varnothing$.

Remark 2.17. (a) Theorems 2.1-2.11 improve and generalize [13, Theorem 6] due to Jungck and Sessa, [18, Theorems 2.2-2.5] of Latif and Tweddle, [26, Theorem 3] of Shahzad, [28, Theorem 4] of Shahzad, [16, Theorem 3.2] of Lami Dozo, and [4, Theorems 1 and 2] of Dotson.

(b) The inequality (2.1) for single-valued maps has been considered by Shahzad [28, 30].

\section{Applications}

3.1. Deterministic fixed point theory. We obtain the following improvements and generalizations of [3, Theorem 2.4], [6, Theorem 3.2], and [34, Theorem 2] and many other results in the current literature.

Theorem 3.1. Let $M$ be a nonempty subset of a normed space $X$ and let $T: M \rightarrow K(M)$ be $a *$-nonexpansive mapping. If $M$ has property $(N)$, then $T$ has a fixed point provided one of the following conditions is satisfied:

(i) $M$ is weakly closed, $X$ is complete space satisfying Opial's condition, and $T(M) \subset B$, for some weakly compact set $B$ in $X$;

(ii) $M$ is weakly compact, $X$ is complete, and $(I-T)$ is demiclosed at 0 ;

(iii) $M$ is weakly compact and $X$ is complete space satisfying Opial's condition;

(iv) $M$ is complete, $T(M)$ is bounded, and $T$ satisfies condition $(A)$;

(v) $M$ is complete, $T(M)$ is bounded, and $T$ is hemicompact;

(vi) $M$ is complete, $T(M)$ is bounded, and $T$ is condensing;

(vii) $M$ is complete, $T(M)$ is bounded, and $T$ satisfies for all $x, y \in M$,

$$
H^{r}(T x, T y) \leq \theta_{1}(d(x, T x)) d^{r}(x, T x)+\theta_{2}(d(y, T y)) d^{r}(y, T y)
$$

where $\theta_{i}: \mathbb{R} \rightarrow[0,1)(i=1,2)$ and $r$ is some fixed positive real number.

Proof. The operator $P_{T}: M \rightarrow K(M)$ is compact-valued and nonexpansive (see, [3, 34]). Further, $\left(1-k_{n}\right) q+k_{n} P_{T} x \subseteq\left(1-k_{n}\right) q+k_{n} T x \subseteq M$, for some $q \in M$ and a fixed sequence of real numbers $k_{n}\left(0 \leq k_{n} \leq 1\right)$ converging to 1 and for each $x \in M$. Thus $M$ has property $(N)$ with respect to $P_{T}$ as $M$ has property $(N)$ with respect to $T$. Also, for each $x \in M$, we 
have, by the definition of $P_{T}$,

$$
d\left(x, P_{T}(x)\right) \leq d\left(x, u_{x}\right)=d(x, T(x)) \leq d\left(x, P_{T}(x)\right) .
$$

(i) As in the proof of [15, Theorem 3.7], $\left(I-P_{T}\right)(M)$ is closed. Now the result follows from Corollary 2.2.

(ii) Suppose that $x_{n} \rightarrow x_{0}$ weakly and $y_{n} \in I-P_{T}\left(x_{n}\right)$ such that $y_{n} \rightarrow 0$ strongly. Note that $y_{n} \in\left(I-P_{T}\right)\left(x_{n}\right) \subseteq(I-T)\left(x_{n}\right)$ and $I-T$ is demiclosed at 0 , so $0 \in(I-T)\left(x_{0}\right)$. This implies that $x_{0} \in T\left(x_{0}\right)$ and hence $d\left(x_{0}, T\left(x_{0}\right)\right)=0$. By $(3.2), d\left(x_{0}, P_{T}\left(x_{0}\right)\right)=d\left(x_{0}, T\left(x_{0}\right)\right)$. Thus $x_{0} \in P_{T}\left(x_{0}\right)$ implies that $I-P_{T}$ is demiclosed at 0 . By Corollary 2.5(a), $P_{T}$ has a fixed point which is also a fixed point of $T$.

(iii) Clearly $\left(I-P_{T}\right)$ is demiclosed at 0 and the result follows from (ii).

On the basis of (3.2), it is easy to prove that if $T$ satisfies the condition $(A)$, then $P_{T}$ satisfies the condition $(A)$, Also if $T$ is hemicompact (condensing), then so is $P_{T}$ (for details, see $[3,15])$. Thus (iv), (v), and (vi) follow from Corollaries 2.8 and 2.9, respectively.

(vii) As in Theorem 2.1, $d\left(x_{n}, P_{T} x_{n}\right) \rightarrow 0$ as $n \rightarrow \infty$; consequently, $\left\{x_{n}\right\}$ is an asymptotically $P_{T}$-regular sequence in $M$. On the basis of (3.2), $d\left(x_{n}, T x_{n}\right) \rightarrow 0$. Thus $\left\{x_{n}\right\}$ is an asymptotically $T$-regular sequence in $M$. The result now follows from Theorem 2.11 .

As noted by $\mathrm{Xu}$, in [34, Proposition 1], that each $f$-nonexpansive-type multivalued map (also called weakly $f$-nonexpansive) is $f$-nonexpansive; consequently, from Corollary 2.5, we obtain the following generalizations of Liu et al. [19, Theorem 4.1 and Corollary 4.2] to a more general class of noncommuting mappings, namely, $T$-weakly commuting maps defined on nonconvex domain which in turn improve and extend [6, Theorems 2.2 and 3.2] and [7, Theorem 3.4] (see also [19, Remark 4.3]).

TheOrem 3.2. Let $f$ be a selfmap on a nonempty weakly compact subset $M$ of a Banach space $X$. Assume that $T: M \rightarrow C(M)$ is $f$-nonexpansive type map such that $M=f(M)$ and $M$ has the property $(N)$. Then $C(f, T) \neq \varnothing$ provided one of the following two conditions is satisfied:

(a) $(f-T)$ is demiclosed at 0 ;

(b) $f$ is weakly continuous, $T$ is compact-valued, and $X$ satisfies Opial's condition.

If, in addition, $f$ is T-weakly commuting at $v$ and $f f v=f v$ for $v \in C(f, T)$, then $F(f) \cap$ $F(T) \neq \varnothing$.

A continuous affine map on a starshaped domain is weakly continuous [4], so we obtain.

COROLlARY 3.3. Let $f$ be a selfmap on a nonempty weakly compact q-starshaped subset $M$ of a Banach space $X$. Assume that $T: M \rightarrow C(M)$ is $f$-nonexpansive-type map such that $M=f(M)$. Then $C(f, T) \neq \varnothing$ provided one of the following two conditions is satisfied:

(a) $(f-T)$ is demiclosed at 0 ;

(b) $f$ is continuous and affine, $T$ is compact-valued, and $X$ satisfies Opial's condition.

If, in addition, $f$ is T-weakly commuting at $v$ and $f f v=f v$ for $v \in C(f, T)$, then $F(f) \cap$ $F(T) \neq \varnothing$. 
A. R. Khan et al. 11

If $T$ is single valued in Theorems 2.1-2.11, $T_{\lambda} x=(1-\lambda) q+\lambda T x \in\{(1-k) q+k T x$ : $0 \leq k \leq 1\}=[q, T x]$. Thus we obtain the following extensions of recent results of Shahzad $[24-26,28,30]$ to the more general class of maps.

Theorem 3.4. Let $T$ and $f$ be selfmaps on a subset $M$ of a normed space $X$. Assume that $M$ has the property $(N)$ (or $M$ is q-starshaped), $M=f(M)$, and $T$ and $f$ satisfy for all $x$, $y \in M$,

$$
\begin{gathered}
\|T x-T y\| \leq \max \{\|f x-f y\|, \operatorname{dist}(f x,[q, T x]), \operatorname{dist}(f y,[q, T y]), \\
\left.\frac{1}{2}[\operatorname{dist}(f x,[q, T y])+\operatorname{dist}(f y,[q, T x])]\right\} .
\end{gathered}
$$

Then $C(f, T) \neq \varnothing$ provided one of the following conditions is satisfied:

(i) $M$ is complete, $T(M)$ is bounded, and $(f-T)(M)$ is closed;

(ii) $M$ is weakly compact, $(f-T)$ is demiclosed at 0 , and $X$ is complete;

(iii) $M$ is complete, $f$ and $T$ satisfy condition $\left(A^{0}\right)$, and $T(M)$ is bounded;

(iv) $M$ is compact and $f$ and $T$ are continuous;

(v) $M$ is complete, $T(M)$ is bounded, and $T$ and $f$ satisfy for all $x, y \in M$,

$$
d^{r}(T x, T y) \leq \theta_{1}(d(f x, T x)) d^{r}(f x, T x)+\theta_{2}(d(f y, T y)) d^{r}(f y, T y),
$$

where $\theta_{i}: \mathbb{R} \rightarrow[0,1)(i=1,2)$ and $r$ is some fixed positive real number.

If, in addition, $f$ is T-weakly commuting at $v$ and $f f v=f v$ for $v \in C(f, T)$, then $F(f) \cap F(T) \neq \varnothing$.

As an application of Theorem 3.4 and Theorem 1.6, we obtain the following common fixed point theorem for a pair of compatible maps under different conditions.

Theorem 3.5. Let $T$ and $f$ be selfmaps on a subset $M$ of a normed space $X$. Assume that $M$ has the property $(N)$ (or $M$ is $q$-starshaped), $M=f(M)$, and $T$ and $f$ satisfy inequality (3.3). If $f$ and $T$ are continuous, compatible and $T$ has relatively compact orbits with d.o.d., then $F(T) \cap F(f) \neq \varnothing$ provided one of the following conditions is satisfied:

(i) $M$ is complete, $T(M)$ is bounded, and $(f-T)(M)$ is closed;

(ii) $M$ is weakly compact, $(f-T)$ is demiclosed at 0 , and $X$ is complete;

(iii) $M$ is complete, $f$ and $T$ satisfy the condition $\left(A^{0}\right)$, and $T(M)$ is bounded;

(iv) $M$ is complete, $T(M)$ is bounded, and $T$ and $f$ satisfy for all $x, y \in M$,

$$
d^{r}(T x, T y) \leq \theta_{1}(d(f x, T x)) d^{r}(f x, T x)+\theta_{2}(d(f y, T y)) d^{r}(f y, T y)
$$

where $\theta_{i}: \mathbb{R} \rightarrow[0,1)(i=1,2)$ and $r$ is some fixed positive real number.

Proof. By Theorem 3.4, in each case, $C(f, T) \neq \varnothing$. Hence the pair $\{f, T\}$ is nontrivially compatible. Thus, by Theorem 1.6, $F(T) \cap F(f) \neq \varnothing$.

The following corollary extends [11, Theorem 4.1] and provides a partial answer to the question of Jungck [11]: "What compacta can be substituted for $I=[0,1]$ in Theorem 4.1 to get the validity of the result?" 
Corollary 3.6. Let $T$ and $f$ be selfmaps on a q-starshaped subset $M$ of a normed space $X$ with $M=f(M)$. Assume that $T$ is $f$-nonexpansive, $f$ is continuous and one of the conditions (i)-(iv) in Theorem 3.5 is satisfied. If $f$ and $T$ are compatible and $T$ has relatively compact orbits with d.o.d., then $F(T) \cap F(f) \neq \varnothing$.

3.2. Approximation theory. As an application of our common fixed point results, we obtain the following invariant approximation results which provide substantial generalizations of recent approximation results of Al-Thagafi [2], Habiniak [5], Jungck and Sessa [13], Kamran [14], Latif and Bano [17], Shahzad [24-26, 28, 30], and many others.

Theorem 3.7. Let $M$ be a subset of a normed space $X$ and let $f: X \rightarrow X$ and $T: X \rightarrow C B(X)$ be mappings such that $u=f u$ and $T u=\{u\}$ for some $u \in X$ and $T(\partial M \cap M) \subset M$. Suppose that $f\left(P_{M}(u)\right)=P_{M}(u)$ and $T$ and $f$ satisfy for all $x \in P_{M}(u) \cup\{u\}$ and $\lambda \in[0,1]$,

$$
H(T x, T y) \leq \begin{cases}\|f x-f u\| & \text { if } y=u, \\ \max \left\{\|f x-f y\|, \operatorname{dist}\left(f x, T_{\lambda} x\right), \operatorname{dist}\left(f y, T_{\lambda} y\right),\right. & \\ \left.\frac{1}{2}\left[\operatorname{dist}\left(f x, T_{\lambda} y\right)+\operatorname{dist}\left(f y, T_{\lambda} x\right)\right]\right\} & \text { if } y \in P_{M}(u) .\end{cases}
$$

Then $P_{M}(u)$ is $T$-invariant. Further, assume that $P_{M}(u)$ has the property $(N)$, and one of the following conditions is satisfied:

(i) $P_{M}(u)$ is complete and $(f-T)\left(P_{M}(u)\right)$ is closed;

(ii) $P_{M}(u)$ is weakly compact, $X$ is complete, and $(f-T)$ is demiclosed at zero;

(iii) $P_{M}(u)$ is complete and $f$ and $T$ satisfy the condition $\left(A^{0}\right)$;

(iv) $P_{M}(u)$ is compact and $f$ and $T$ are continuous on $P_{M}(u)$;

(v) $P_{M}(u)$ is complete and $T$ and $f$ satisfy for all $x, y \in M$,

$$
H^{r}(T x, T y) \leq \theta_{1}(d(f x, T x)) d^{r}(f x, T x)+\theta_{2}(d(f y, T y)) d^{r}(f y, T y),
$$

where $\theta_{i}: \mathbb{R} \rightarrow[0,1)(i=1,2)$ and $r$ is some fixed positive real number.

Then $P_{M}(u) \cap C(f, T) \neq \varnothing$. If, in addition, $f$ is $T$-weakly commuting at $v$ and $f f v=$ fv for $v \in C(f, T)$, then $P_{M}(u) \cap F(f) \cap F(T) \neq \varnothing$.

Proof. Let $x \in P_{M}(u)$. Then $f x \in P_{M}(u)$ since $f\left(P_{M}(u)\right)=P_{M}(u)$. By the definition of $P_{M}(u), x \in \partial M \cap M$ and since $T(\partial M \cap M) \subset M$, it follows that $T x \subset M$. Let $z \in T(x)$. Then, by (3.6),

$$
d(z, u) \leq H(T x, T u) \leq d(f x, f u)=d(f x, u)=\operatorname{dist}(u, M) .
$$

Now $z \in M$ and $f(x) \in P_{M}(u)$ imply that $z \in P_{M}(u)$. Thus $T(x) \subset P_{M}(u)$. Hence $T$ maps $P_{M}(u)$ into $C B\left(P_{M}(u)\right)$. Now if (i) holds, then the result follows from Theorem 2.1. In case (ii), (iii), (iv), or (v) holds, result follows from Theorem 2.4, 2.6, 2.10, or 2.11, respectively.

As an application of Corollaries 2.3, 2.5, and 2.7 and Theorems 2.10 and 2.11, we immediately obtain the following result which improves and generalizes $[14$, Theorem 
3.14] due to Kamran and extends [13, Theorem 7] due to Jungck and Sessa, and contains all of the results of Latif and Bano [17] as special cases.

Theorem 3.8. Let $M$ be a subset of a normed space $X, f: X \rightarrow X$ and $T: X \rightarrow C B(X)$. Assume that $P_{M}(u)$ is nonempty $q$-starshaped with $f\left(P_{M}(u)\right)=P_{M}(u)$ and $T$ is $f$-nonexpansive on $P_{M}(u)$. Suppose that $P_{M}(u)$ is $T$-invariant and one of the following conditions is satisfied:

(i) $P_{M}(u)$ is complete and $(f-T)\left(P_{M}(u)\right)$ is closed;

(ii) $P_{M}(u)$ is weakly compact, $X$ is complete, and $(f-T)$ is demiclosed at 0 ;

(iii) $P_{M}(u)$ is weakly compact, $f$ is weakly continuous, and $X$ is Banach space satisfying Opial's condition;

(iv) $P_{M}(u)$ is complete and $f$ and $T$ satisfy the condition $\left(A^{0}\right)$;

(v) $P_{M}(u)$ is compact and $f$ is continuous on $P_{M}(u)$;

(vi) $P_{M}(u)$ is complete and $T$ and $f$ satisfy for all $x, y \in M$,

$$
H^{r}(T x, T y) \leq \theta_{1}(d(f x, T x)) d^{r}(f x, T x)+\theta_{2}(d(f y, T y)) d^{r}(f y, T y)
$$

where $\theta_{i}: \mathbb{R} \rightarrow[0,1)(i=1,2)$ and $r$ is some fixed positive real number. Then $P_{M}(u) \cap$ $C(f, T) \neq \varnothing$. If, in addition, $f$ is $T$-weakly commuting at $v$ and $f f v=f v$ for $v \in C(f, T)$, then $P_{M}(u) \cap F(f) \cap F(T) \neq \varnothing$.

Remark 3.9. (a) Theorem 3.7 illustrates that the conclusion of recent invariant approximation result of Kamran [14, Theorem 3.14] holds for generalized $f$-nonexpansive $T$ weakly commuting maps $f$ where $P_{M}(u)$ need not be compact and starshaped.

(b) Theorem 3.8 contains Theorems 1.1 and 1.2 and many others.

(c) It is obvious from Theorem 3.8(v) that [14, Theorem 3.14] of Kamran holds without the conditions;

(i) $u \in F(f) \cap F(T), f$ is affine and $f q=q$;

(ii) $f$ and $A_{\lambda}$ satisfy the property $(E . A)$ for each $\lambda \in[0,1]$ where $A_{\lambda}(x)=(1-\lambda) p+$ $\lambda T x$.

If $T$ is single valued in Theorem 3.7, we obtain the following result which shows validity of [24, Theorem 6], [26, Theorem 4], and [28, Theorem 5] for T-weakly commuting and compatible maps where $f$ need not be affine and weakly continuous.

Corollary 3.10. Let $M$ be a subset of a normed space $X, f, T: X \rightarrow X$ be mappings such that $u=f u=T u$ for some $u \in X$ and $T(\partial M \cap M) \subset M$. Suppose that $P_{M}(u)$ is $q-$ starshaped, $f\left(P_{M}(u)\right)=P_{M}(u)$ and $T$ and $f$ satisfy for all $x \in P_{M}(u) \cup\{u\}$,

$$
\|T x-T y\| \leq \begin{cases}\|f x-f u\| & \text { if } y=u, \\ \max \{\|f x-f y\|, \operatorname{dist}(f x,[q, T x]), \operatorname{dist}(f y,[q, T y]), & \\ \left.\frac{1}{2}[\operatorname{dist}(f x,[q, T y])+\operatorname{dist}(f y,[q, T x])]\right\} & \text { if } y \in P_{M}(u) .\end{cases}
$$


Assume that one of the conditions (i)-(v) in Theorem 3.8 holds. Then $P_{M}(u) \cap C(f, T) \neq$ $\varnothing$. If, in addition, $f$ is T-weakly commuting at $v$ and $f f v=f v$ for $v \in C(f, T)$, then $P_{M}(u) \cap F(f) \cap F(T) \neq \varnothing$.

Corollary 3.11. Let $M$ be a subset of a normed space $X$ and let $f, T: X \rightarrow X$ be mappings such that $u=f u=T u$ for some $u \in X$ and $T(\partial M \cap M) \subset M$. Suppose that $f\left(P_{M}(u)\right)=$ $P_{M}(u)$, T and $f$ satisfy (3.10) for all $x \in P_{M}(u) \cup\{u\}, P_{M}(u)$ is q-starshaped, and one of the conditions (i)-(iv) in Theorem 3.5 holds. If $f$ and $T$ are continuous and compatible and $T$ has relatively compact orbits with d.o.d., then $P_{M}(u) \cap F(f) \cap F(T) \neq \varnothing$.

3.3. Random fixed point theory. Throughout this section, $\left(\Omega, \sum\right)$ denotes a measurable space. A mapping $T: \Omega \rightarrow C B(X)$ is called measurable if for any open subset $M$ of $X$, $T^{-1}(C)=\{\omega \in \Omega: T(\omega) \cap M \neq \phi\} \in \sum$. A mapping $T: \Omega \times X \rightarrow C B(X)(f: \Omega \times X \rightarrow X)$ is called a random operator if for any $x \in X, T(\cdot, x)(f(\cdot, x))$ is measurable. A mapping $T: \Omega \times X \rightarrow K(X)$ is called a random operator in the sense of Gorniewicz operator [1] if $T$ is product measurable and $T(\omega, \cdot)$ is upper semicontinuous for every $\omega \in \Omega$. A measurable mapping $\xi: \Omega \rightarrow X$ is called a random fixed point of a random operator $T: \Omega \times X \rightarrow C(X)$ if, for every $\omega \in \Omega, \xi(\omega) \in T(\omega, \xi(\omega))$. A measurable mapping $\xi: \Omega \rightarrow X$ is a random coincidence point of random operators $T: \Omega \times X \rightarrow C(X)$ and $f: \Omega \times X \rightarrow X$ if for every $\omega \in \Omega, f(\omega, \xi(\omega)) \in T(\omega, \xi(\omega))$ (for details, see [29, 31, 33]).

If we combine [1, Theorem 1.3] and Theorem 3.1, then we have the following random fixed point theorem for a noncontinuous class of maps (see also [3]) which generalizes the corresponding results in $[3,6,15]$.

Theorem 3.12. Let $\left(\Omega, \sum\right)$ be a complete measurable space and $M$ a nonempty subset of a separable Banach space X. Suppose that $T: \Omega \times M \rightarrow K(M)$ is $*$-nonexpansive and random in the sense of Gorniewicz. Assume that $M$ has the property $(N)$ (or $M$ is q-starshaped) and one of the following conditions is satisfied:

(i) $M$ is weakly closed, $X$ satisfies Opial's condition and for each $\omega \in \Omega, T(\omega, M) \subset B$, for some weakly compact set $B$ in $X$;

(ii) $M$ is weakly compact and for each $\omega \in \Omega,(I-T)(\omega, \cdot)$ is demiclosed at 0 ;

(iii) $M$ is weakly compact and $X$ satisfies Opial's condition;

(iv) $M$ is closed, $T(\omega, M)$ is bounded, and $T(\omega, \cdot)$ satisfies the condition $(A)$, for each $\omega \in \Omega$;

(v) $M$ is closed, $T(\omega, M)$ is bounded, and $T(\omega, \cdot)$ is hemicompact, for each $\omega \in \Omega$;

(vi) $M$ is closed, $T(\omega, M)$ is bounded, and $T(\omega, \cdot)$ is condensing, for each $\omega \in \Omega$;

(vii) $M$ is closed, $T(\omega, M)$ is bounded, and $T(\omega, \cdot)$ satisfies for each $\omega \in \Omega$ and $x, y \in M$,

$$
\begin{aligned}
H^{r}(T(\omega, x), T(\omega, y)) \leq & \theta_{1}(d(x, T(\omega, x))) d^{r}(x, T(\omega, x)) \\
& +\theta_{2}(d(y, T(\omega, y))) d^{r}(y, T(\omega, y)),
\end{aligned}
$$

where $\theta_{i}: \mathbb{R} \rightarrow[0,1)(i=1,2)$ and $r$ is some fixed positive real number. Then $T$ has a random fixed point.

If we combine Theorem 2.4 (or Corollary 2.5) and Theorem 1.7, we obtain the following results which generalize and improve [31, Theorems 3.2 and 3.3] due to Shahzad 
and Latif and [33, Theorem 1] of Xu, in the sense that the maps $f$ and $T$ need not be commuting for the existence of random coincidence, $T(\omega, \cdot)$ is not necessarily $f(\omega, \cdot)$ nonexpansive, and $f$ is not affine.

Theorem 3.13. Let $\left(\Omega, \sum\right)$ be any measurable space, $M$ a nonempty separable weakly compact subset of a Banach space $X$, and $f: \Omega \times M \rightarrow M$ a random operator such that $f(\omega, M)$ $=M$ for each $\omega \in \Omega$. Assume that $T: \Omega \times M \rightarrow C B(M)$ is continuous and satisfies, for all $\omega \in \Omega, x, y \in M$, and $\lambda \in[0,1]$,

$$
\begin{aligned}
& H(T(\omega, x), T(\omega, y)) \\
& \leq \max \left\{\|f(\omega, x)-f(\omega, y)\|, \operatorname{dist}\left(f(\omega, x), T_{\lambda}(\omega, x)\right), \operatorname{dist}\left(f(\omega, y), T_{\lambda}(\omega, y)\right),\right. \\
& \left.\frac{1}{2}\left[\operatorname{dist}\left(f(\omega, x), T_{\lambda}(\omega, y)\right)+\operatorname{dist}\left(f(\omega, y), T_{\lambda}(\omega, x)\right)\right]\right\} .
\end{aligned}
$$

Suppose that $M$ has property $(N)$ (or $M$ is q-starshaped), $f$ is both continuous and weakly continuous, and $(f-T)(\omega, \cdot)$ is demiclosed at 0 for each $\omega \in \Omega$. Then $f$ and $T$ have $a$ random coincidence point. Moreover, if for each $\omega \in \Omega$ and any $x \in M, f(\omega, x) \in T(\omega, x)$ implies $f(\omega, f(\omega, x))=f(\omega, x)$, and $f$ is T-weakly commuting random operator, then $f$ and $T$ have a common random fixed point.

Corollary 3.14. Let $\left(\Omega, \sum\right)$ be any measurable space and $M$ a nonempty separable weakly compact subset of a Banach space $X$. Assume that $T: \Omega \times M \rightarrow C B(M)$ satisfies, for all $\omega \in \Omega, x, y \in M$, and $\lambda \in[0,1]$,

$$
\begin{aligned}
H(T(\omega, x), T(\omega, y)) \leq \max \{ & \|x-y\|, \operatorname{dist}\left(x, T_{\lambda}(\omega, x)\right), \operatorname{dist}\left(y, T_{\lambda}(\omega, y)\right), \\
& \left.\frac{1}{2}\left[\operatorname{dist}\left(x, T_{\lambda}(\omega, y)\right)+\operatorname{dist}\left(y, T_{\lambda}(\omega, x)\right)\right]\right\} .
\end{aligned}
$$

Suppose that $M$ has property $(N)$ (or $M$ is q-starshaped) and $(I-T)(\omega, \cdot)$ is demiclosed at 0 for each $\omega \in \Omega$. Then $T$ has a random fixed point.

Corollary 3.15. Let $\left(\Omega, \sum\right)$ be any measurable space, let $M$ be a separable weakly compact subset of a Banach space $X$ which is q-starshaped, and let $f: \Omega \times M \rightarrow M$ be a continuous affine random operator such that $f(\omega, M)=M$ for each $\omega \in \Omega$. Let $T: \Omega \times M \rightarrow C B(M)$ be an $f$-nonexpansive random operator. If one of the following conditions is satisfied:

(a) $(f-T)(\omega, \cdot)$ is demiclosed at 0 for each $\omega \in \Omega$;

(b) $T(\omega, \cdot)$ is compact-valued for each $\omega \in \Omega$ and $X$ satisfies Opial's condition, then $f$ and T have a random coincidence point. Moreover, if for each $\omega \in \Omega$ and any $x \in M$, $f(\omega, x) \in T(\omega, x)$ implies $f(\omega, f(\omega, x))=f(\omega, x)$, and $f$ is T-weakly commuting random operator, then $f$ and $T$ have a common random fixed point.

If we combine Theorem 2.6 (or Corollary 2.7) and Theorem 1.8, we obtain the conclusion of [29, Theorem 3.18], without the commutativity of maps. Notice that $f$ need not be affine and $f(\omega, \cdot)$ need not fix $q$ for each $\omega \in \Omega$. 
Theorem 3.16. Let $\left(\Omega, \sum\right)$ be any measurable space, $M$ a nonempty separable closed subset of a Banach space $X$, and $f: \Omega \times M \rightarrow M$ a continuous random operator such that $f(\omega, M)=M$ for each $\omega \in \Omega$. Assume that the random operator $T: \Omega \times M \rightarrow C B(M)$ is continuous and satisfies (3.12) (or is $f$-nonexpansive). If $M$ is q-starshaped, $f$ and $T$ satisfy the condition $\left(A^{0}\right)$ and $T(\omega, M)$ is bounded for each $\omega \in \Omega$, then $f$ and $T$ have a random coincidence point. Moreover, if for each $\omega \in \Omega$ and any $x \in M, f(\omega, x) \in T(\omega, x)$ implies $f(\omega, f(\omega, x))=f(\omega, x)$, and $f$ is T-weakly commuting random operator, then $f$ and $T$ have a common random fixed point.

Remark 3.17. (a) If we combine Theorems 2.10 and 1.8 , we obtain the conclusion of [29, Theorem 3.17], without the commutativity of maps. Notice that $f$ need not be affine and $f(\omega, \cdot)$ need not fix $q$ for each $\omega \in \Omega$.

(b) Using Theorems 3.13-3.16, we can obtain the random invariant approximation results. In particular, when $T$ is single-valued, we obtain the following result which provides stochastic analogue (for more general maps) of the recent invariant approximation result due to Shahzad [28, Theorem 5].

Corollary 3.18. Let $M$ be a subset of a Banach space $X$ and let $f, T: \Omega \times X \rightarrow X$ be random operators such that $u=f(\omega, u)=T(\omega, u)$ for each $\omega \in \Omega$ and for some $u \in X$. Suppose that $P_{M}(u)$ is nonempty and $q$-starshaped, $f(\omega, \cdot)$ and $T(\omega, \cdot)$ are continuous on $P_{M}(u)$, $f\left(\omega, P_{M}(u)\right)=P_{M}(u), T(\omega, \partial M \cap M) \subset M$ for each $\omega \in \Omega$, and $T(\omega, \cdot)$ and $f(\omega, \cdot)$ satisfy (3.10) for each $\omega \in \Omega$ and $x \in P_{M}(u) \cup\{u\}$. Suppose that one of the following conditions is satisfied:

(i) $P_{M}(u)$ is separable weakly compact, $f$ is weakly continuous, and $(f-T)(\omega, \cdot)$ is demiclosed at 0 for each $\omega \in \Omega$;

(ii) $P_{M}(u)$ is separable closed and $f(\omega, \cdot)$ and $T(\omega, \cdot)$ satisfy the condition $\left(A^{0}\right)$ for each $\omega \in \Omega$.

Then $P_{M}(u) \cap C(f, T) \neq \varnothing$. If, in addition, for each $\omega \in \Omega$ and any $x \in M, f(\omega, x)=$ $T(\omega, x)$ implies $f(\omega, f(\omega, x))=f(\omega, x)$, and $f$ is T-weakly commuting random operator, then $P_{M}(u) \cap F(f) \cap F(T) \neq \varnothing$.

\section{Acknowledgments}

The authors are grateful to Professors G. Jungck and N. Shahzad for providing preprints of $[12,30]$, respectively. The authors would like to thank the referees for their valuable suggestions to improve the presentation of the paper. The first author gratefully acknowledges support provided by King Fahd University of Petroleum \& Minerals during this research.

\section{References}

[1] R. P. Agarwal, D. O’Regan, and M. Sambandham, Random degree and essentiality for countably condensing maps, Stochastic Analysis and Applications 20 (2002), no. 6, 1169-1176.

[2] M. A. Al-Thagafi, Common fixed points and best approximation, Journal of Approximation Theory 85 (1996), no. 3, 318-323.

[3] I. Beg, A. R. Khan, and N. Hussain, Approximation of $*$-nonexpansive random multivalued operators on Banach spaces, Journal of the Australian Mathematical Society 76 (2004), no. 1, 51-66. 
[4] W. G. Dotson Jr., Fixed point theorems for non-expansive mappings on star-shaped subsets of Banach spaces, Journal of the London Mathematical Society. Second Series 4 (1972), 408-410.

[5] L. Habiniak, Fixed point theorems and invariant approximations, Journal of Approximation Theory 56 (1989), no. 3, 241-244.

[6] T. Husain and A. Latif, Fixed points of multivalued nonexpansive maps, Mathematica Japonica 33 (1988), no. 3, 385-391.

[7] __ Fixed points of multivalued nonexpansive maps, International Journal of Mathematics and Mathematical Sciences 14 (1991), no. 3, 421-430.

[8] N. Hussain, Coincidence points for multivalued maps defined on non-starshaped domain, to appear in Demonstratio Mathematica.

[9] N. Hussain and A. R. Khan, Common fixed-point results in best approximation theory, Applied Mathematics Letters 16 (2003), no. 4, 575-580.

[10] N. Hussain, D. O’Regan, and R. P. Agarwal, Common fixed point and invariant approximation results on non-starshaped domain, Georgian Mathematical Journal 12 (2005), 659-669.

[11] G. Jungck, Coincidence and fixed points for compatible and relatively nonexpansive maps, International Journal of Mathematics and Mathematical Sciences 16 (1993), no. 1, 95-100.

[12] Common fixed point theorems for compatible self maps of Hausdorff topological spaces, Fixed Point Theory and Applications 2005 (2005), no. 3, 355-363.

[13] G. Jungck and S. Sessa, Fixed point theorems in best approximation theory, Mathematica Japonica 42 (1995), no. 2, 249-252.

[14] T. Kamran, Coincidence and fixed points for hybrid strict contractions, Journal of Mathematical Analysis and Applications 299 (2004), no. 1, 235-241.

[15] A. R. Khan and N. Hussain, Random fixed points for $*$-nonexpansive random operators, Journal of Applied Mathematics and Stochastic Analysis 14 (2001), no. 4, 341-349.

[16] E. Lami Dozo, Multivalued nonexpansive mappings and Opial's condition, Proceedings of the American Mathematical Society 38 (1973), 286-292.

[17] A. Latif and A. Bano, A result on invariant approximation, Tamkang Journal of Mathematics 33 (2002), no. 1, 89-92.

[18] A. Latif and I. Tweddle, On multivalued $f$-nonexpansive maps, Demonstratio Mathematica 32 (1999), no. 3, 565-574.

[19] Z. Liu, J. S. Ume, and M. S. Khan, Coincidence and fixed point theorems in metric and Banach spaces, International Journal of Mathematics and Mathematical Sciences 26 (2001), no. 6, 331339.

[20] G. Meinardus, Invarianz bei linearen approximationen, Archive for Rational Mechanics and Analysis 14 (1963), 301-303.

[21] S. A. Naimpally, K. L. Singh, and J. H. M. Whitfield, Fixed points and nonexpansive retracts in locally convex spaces, Fundamenta Mathematicae 120 (1984), no. 1, 63-75.

[22] H. K. Pathak and M. S. Khan, Fixed and coincidence points of hybrid mappings, Archivum Mathematicum (Brno) 38 (2002), no. 3, 201-208.

[23] S. A. Sahab, M. S. Khan, and S. Sessa, A result in best approximation theory, Journal of Approximation Theory 55 (1988), no. 3, 349-351.

[24] N. Shahzad, A result on best approximation, Tamkang Journal of Mathematics 29 (1998), no. 3, 223-226.

[25] _ Correction to: "A result on best approximation", Tamkang Journal of Mathematics 30 (1999), no. 2, 165.

[26] _ On R-subcommuting maps and best approximations in Banach spaces, Tamkang Journal of Mathematics 32 (2001), no. 1, 51-53. 
18 Coincidences and approximations of generalized $f$-nonexpansive maps

[27] Coincidence points and R-subweakly commuting multivalued maps, Demonstratio Mathematica 36 (2003), no. 2, 427-431.

[28] _ Generalized I-nonexpansive maps and best approximations in Banach spaces, Demonstratio Mathematica 37 (2004), no. 3, 597-600.

[29] Some general random coincidence point theorems, New Zealand Journal of Mathematics 33 (2004), no. 1, 95-103.

[30] _ Invariant approximations, generalized I-contractions, and R-subweakly commuting maps, Fixed Point Theory and Applications 2005 (2005), no. 1, 79-86.

[31] N. Shahzad and A. Latif, A random coincidence point theorem, Journal of Mathematical Analysis and Applications 245 (2000), no. 2, 633-638.

[32] S. P. Singh, An application of a fixed-point theorem to approximation theory, Journal of Approximation Theory 25 (1979), no. 1, 89-90.

[33] H. K. Xu, Some random fixed point theorems for condensing and nonexpansive operators, Proceedings of the American Mathematical Society 110 (1990), no. 2, 395-400.

[34] Japonica 36 (1991), no. 3, 441-445.

A. R. Khan: Department of Mathematical Sciences, King Fahd University of Petroleum \& Minerals, Dhahran, Saudi Arabia

E-mail address: arahim@kfupm.edu.sa

F. Akbar: Department of Mathematics, University of Sargodha, Sargodha 40100, Pakistan

E-mail address:ridaf75@yahoo.com

N. Sultana: Department of Mathematics, University of Sargodha, Sargodha 40100, Pakistan

E-mail address: pdnaz@yahoo.com

N. Hussain: Department of Mathematics, King Abdul Aziz University, P. O. Box 80203, Jeddah 21589, Saudi Arabia

E-mail address: nhabdullah@kau.edu.sa 


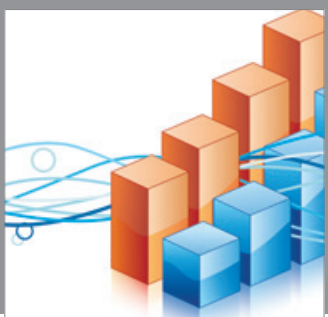

Advances in

Operations Research

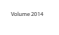

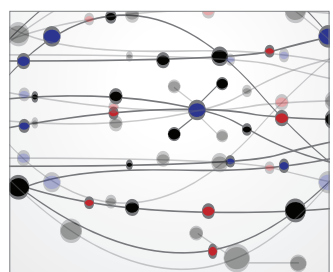

\section{The Scientific} World Journal
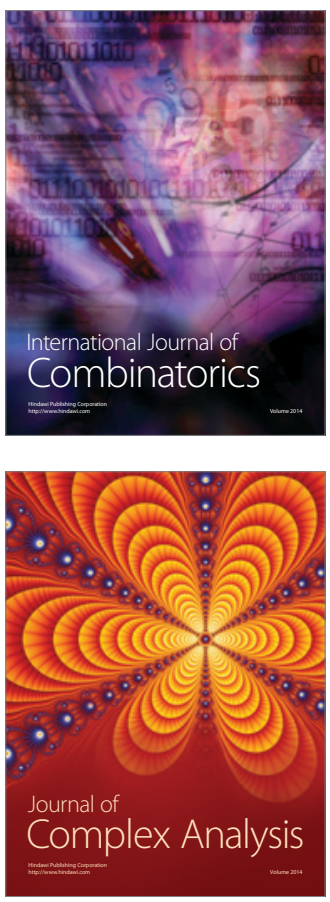

International Journal of

Mathematics and

Mathematical

Sciences
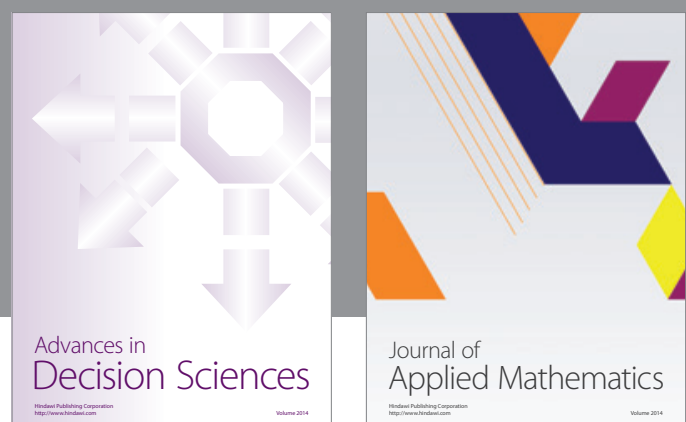

Journal of

Applied Mathematics
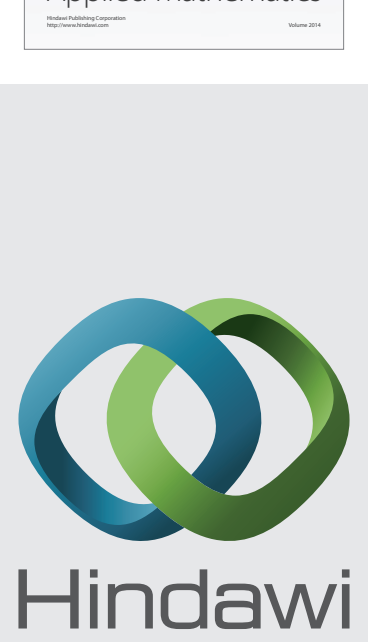

Submit your manuscripts at http://www.hindawi.com
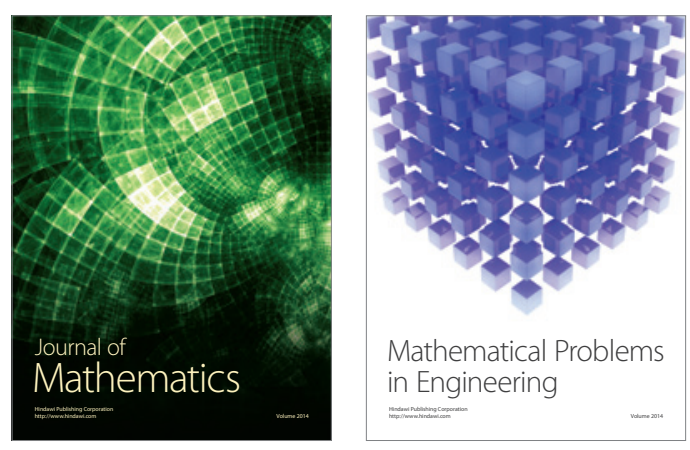

Mathematical Problems in Engineering
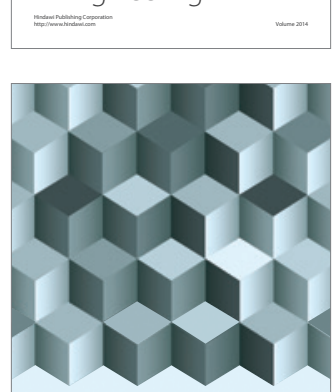

Journal of

Function Spaces
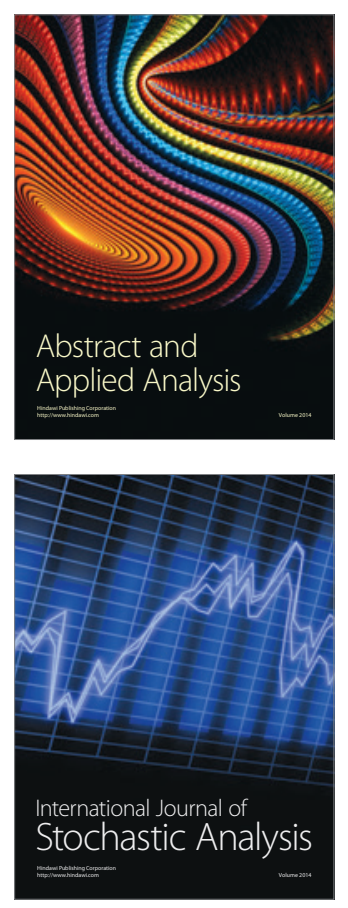

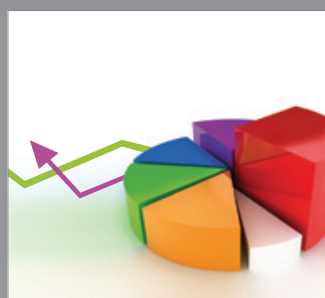

ournal of

Probability and Statistics

Promensencen
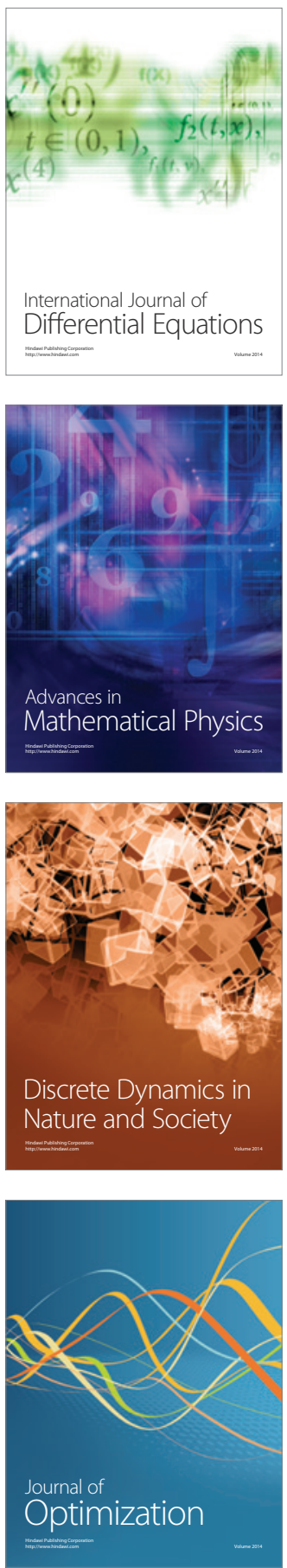